PAPER

\title{
Is it worth pursuing surgery for epilepsy in patients with normal neuroimaging?
}

\section{G Alarcón, A Valentín, C Watt, R P Selway, M E Lacruz, R D C Elwes, J M Jarosz, M Honavar, F Brunhuber, N Mullatti, I Bodi, M Salinas, C D Binnie, C E Polkey}

See Editorial Commentary, p 428

J Neurol Neurosurg Psychiatry 2006;77:474-480. doi: 10.1136/jnnp.2005.077289

Objective: To determine whether it is worth pursuing surgery for the treatment of epilepsy in patients with normal neuroimaging.

Methods: Two patient populations were studied: (1) 136 consecutive patients who were surgically treated; (2) 105 consecutive patients assessed with chronically implanted intracranial electrodes within the same period. Sixty patients belonged to both groups, and included all 21 patients who had normal neuroimaging.

See end of article for authors' affiliations

Results: There were no differences in the proportion of patients with favourable outcome between those with normal and those with abnormal neuroimaging, irrespective of whether intracranial recordings were required. Among the 19 operated patients with normal neuroimaging, 74\% had a favourable outcome Correspondence to: Dr Gonzalo Alarcón, Department of Clinical Neurophysiology, King's College Hospital,' Denmark Hill, London SE5 9RS, UK; galarcon@aol.com

Received 9 August 2005

In revised form

25 October 2005

Accepted

30 November 2005 (Engel's seizure outcome grades I and II), and among the 93 patients with abnormal neuroimaging, 73\% had favourable outcome $(p=0.96)$. In patients with temporal resections, $92 \%$ of the 13 patients with normal neuroimaging had a favourable outcome, whereas among the 70 patients with abnormal neuroimaging, $80 \%$ had a favourable outcome $(p=0.44)$. In patients with extratemporal resections, two of the six patients with normal neuroimaging had a favourable outcome, while 12 of the 23 patients with abnormal neuroimaging had a favourable outcome $(p=0.65)$. Among the 105 patients studied with intracranial electrodes, five suffered transitory deficits as a result of implantation, and two suffered permanent deficits (one hemiplegia caused by haematoma and one mild dysphasia resulting from haemorrhage).

Conclusions: It is worth pursuing surgery in patients with normal neuroimaging because it results in good seizure control and the incidence of permanent deficits associated with intracranial studies is low.
$\mathrm{P}$ resurgical assessment of patients with epilepsy and normal neuroimaging has long been controversial. Scott et al reported a study of 40 patients with normal magnetic resonance imaging (MRI) assessed between 1995 and 1997. ${ }^{1}$ No patient had a well localised epileptogenic zone concordant with other tests that would have allowed one to proceed directly to surgery. In only five patients did evaluation lead to a hypothesis that could be tested by intracranial studies, and only three proceeded to surgery. An editorial on the same issue suggested that the authors merely stated their practice. ${ }^{2}$ An important question is whether surgical outcome is worse in patients with normal neuroimaging. Several reports on small series of patients have suggested that the majority of patients with normal neuroimaging remain seizure-free or enjoy a more than $90 \%$ reduction in seizures after temporal lobe, ${ }^{34}$, extratemporal $^{4}{ }^{5}$ or peri-rolandic surgery. ${ }^{6}$ A larger study on 115 patients appeared to confirm these findings. ${ }^{7}$ In extratemporal epilepsy, good results have been reported in MRI negative patients, ${ }^{8}$ although the presence of a focal abnormality on neuroimaging appears to be a favourable prognostic sign in frontal lobe epilepsy surgery. ${ }^{10}$ Indeed, modern advances in neuroimaging have reduced the proportion of patients with normal neuroimaging. It is nevertheless unclear whether patients with normal neuroimaging suffer worse seizure control after surgery and, if so, to what degree their seizure control is inferior to that in patients with neuroimaging abnormalities.

The general question of whether it is worth pursuing surgery in patients with normal neuroimaging can be subdivided as follows:
- What proportion of patients assessed for surgery have normal neuroimaging?

- Are intracranial electrodes necessary? If so, what proto$\operatorname{col}(\mathrm{s})$ should be followed?

- Is seizure control adequate after surgery in patients with normal neuroimaging?

- Do the benefits of intracranial electrodes justify their risks?

We have addressed these questions in all those 136 patients operated on and in all 105 patients assessed with chronically implanted intracranial electrodes at King's College Hospital between September 1999 and May 2004. The proportion of patients assessed for surgery who have normal neuroimaging depends on the centre's selection criteria and the stage at which patients are considered to have entered the surgery programme. At our centre, no patients are rejected a priori solely on the basis of having normal neuroimaging. We have chosen two populations of patients who have clearly entered the surgical programme: the operated patients and the patients assessed with intracranial electrodes. Surgical outcome has been compared among patients with normal and abnormal neuroimaging in both patient groups. Among all operated patients, all patients with normal neuroimaging were assessed using intracranial electrodes, whereas not all patients with abnormal neuroimaging had intracranial recordings. Thus differences in outcome among patients with normal and abnormal

Abbreviations: Abbreviation: DNT, dysembryoplastic neuroepithelial tumour; FCD, focal cortical dysplasia 
neuroimaging could reflect differences in presurgical assessment because of the extensive use of intracranial electrodes in patients with normal neuroimaging. In order to minimise this effect, differences in surgical outcome between patients with normal and abnormal neuroimaging have also been studied within the subpopulation of operated patients assessed using intracranial electrodes.

\section{METHODS \\ Subjects}

The study included patients operated on at King's College Hospital between September 1999 and May 2004 for the treatment of medically refractory epilepsy. Four patients who had surgery for Landau-Kleffner syndrome within this period were excluded from the study, as the main aim of surgery in these patients was to improve language rather than the epilepsy. We have studied two patient populations: first, a group of 136 consecutive patients (70 male, 66 female, median age 33 years, age range 2 to 59 years) who were surgically treated for epilepsy at King's College Hospital within the recruiting period; and second, 105 consecutive patients assessed with chronically implanted intracranial electrodes within the same period (52 male, 53 female, median age 31 years, age range 1 to 58 years). Sixty patients belonged to both groups (they were assessed before surgery using chronically implanted intracranial electrodes and were then operated on). All 41 patients who had normal neuroimaging were assessed using intracranial electrodes. Among these 41 patients, 21 had been operated on at the time of analysis and 20 had not. The composition and interrelations of these patient populations can be seen in fig 1 . In patients assessed using chronically implanted intracranial electrodes, the type, number, and position of the electrodes were determined by the location of the suspected epileptogenic zone in each patient, according to findings from clinical history, neuroimaging, neuropsychology, scalp electroencephalographic recordings, and videotelemetry. The selection criteria and implantation procedure have been described elsewhere ${ }^{11}$ and are summarised below.

Among the 136 surgically treated patients, 101 had temporal lobe resections, 32 had extratemporal surgery, and

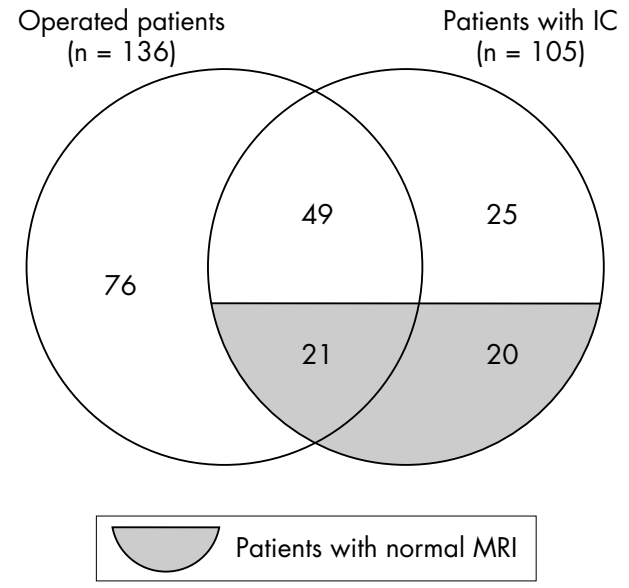

Figure 1 Composition and interrelations of the patient populations involved in the study: 136 patients had been operated on at the time of analysis and 105 had been assessed using intracranial recordings; 60 patients had been assessed with intracranial recordings and operated on. All 41 patients with normal neuroimaging were assessed with intracranial electrodes; of these 21 had been operated on at the time of analysis. IC, assessment with intracranial electrodes.

three had a resection involving the temporal and occipital lobes. One hundred and twelve had a follow up period of at least 12 months at the time of analysis ( 83 temporal, 26 extratemporal, and three temporo-occipital resections). For analysis, the three patients who had temporo-occipital resections were considered as extratemporal.

Among the 105 patients assessed with intracranial electrodes, 63 proved to have temporal lobe epilepsy, 39 extratemporal epilepsy, and in three the source of their epilepsy remained unknown. Of the 105 patients, 60 were operated on (39 had temporal procedures, 19 had extratemporal procedures, and two had a temporo-occipital resection). Among these 60 surgically treated patients, 53 had a follow up period of at least 12 months at the time of analysis (35 temporal, 16 extratemporal, and two

Table 1 Postsurgical seizure control in patients with normal and abnormal neuroimaging and follow up period of at least 12 months

\begin{tabular}{|c|c|c|c|c|c|}
\hline & $\mathbf{n}$ & I & II & III & IV \\
\hline \multicolumn{6}{|c|}{ Temporal and extratemporal procedures } \\
\hline \multicolumn{6}{|c|}{ All operated patients } \\
\hline Normal neuroimaging & 19 & $9(47.4 \%)$ & $5(26.3 \%)$ & $3(15.8 \%)$ & $2(10.5 \%)$ \\
\hline Abnormal neuroimaging & 93 & $47(50.5 \%)$ & $21(22.6 \%)$ & $16(17.2 \%)$ & $9(9.7 \%)$ \\
\hline \multicolumn{6}{|c|}{ Patients with intracranial electrodes } \\
\hline Normal neuroimaging & 19 & $9(47.4 \%)$ & $5(26.3 \%)$ & $3(15.8 \%)$ & $2(10.5 \%)$ \\
\hline Abnormal neuroimaging & 34 & $16(47 \%)$ & $7(20.6 \%)$ & $8(23.5 \%)$ & $3(8.8 \%)$ \\
\hline \multicolumn{6}{|l|}{ Temporal procedures } \\
\hline \multicolumn{6}{|l|}{ All operated patients } \\
\hline Normal neuroimaging & 13 & $8(61.6 \%)$ & $4(30.8 \%)$ & $1(7.7 \%)$ & 0 \\
\hline Abnormal neuroimaging & 70 & $38(54.3 \%)$ & $18(25.7 \%)$ & $8(11.4 \%)$ & $6(8.6 \%)$ \\
\hline \multicolumn{6}{|c|}{ Patients with intracranial electrodes } \\
\hline Normal neuroimaging & 13 & $8(61.6 \%)$ & $4(30.8 \%)$ & $1(7.7 \%)$ & 0 \\
\hline Abnormal neuroimaging & 22 & $12(54.6 \%)$ & $6(27.3 \%)$ & $2(9.1 \%)$ & $2(9.1 \%)$ \\
\hline \multicolumn{6}{|c|}{ Extratemporal procedures (including temporo-occipital resections) } \\
\hline \multicolumn{6}{|c|}{ All operated patients } \\
\hline Normal neuroimaging & 6 & $1(16.7 \%)$ & $1(16.7 \%)$ & $2(33.3 \%)$ & $2(33.3 \%)$ \\
\hline Abnormal neuroimaging & 23 & 9 (39.1\%) & $3(13 \%)$ & $8(34.8 \%)$ & $3(13 \%)$ \\
\hline \multicolumn{6}{|c|}{ Patients with intracranial electrodes } \\
\hline Normal neuroimaging & 6 & $1(16.7 \%)$ & $1(16.7 \%)$ & $2(33.3 \%)$ & $2(33.3 \%)$ \\
\hline Abnormal neuroimaging & 12 & $4(33.3 \%)$ & $1(8.3 \%)$ & $6(50 \%)$ & $1(8.3 \%)$ \\
\hline
\end{tabular}


Table 2 Comparison of postsurgical seizure control in patients with normal and abnormal neuroimaging and follow up period of at least 12 months

\begin{tabular}{|c|c|c|c|c|}
\hline & $\mathbf{n}$ & Favourable outcome (I-II) & Poor outcome (III-IV) & $\chi^{2}, p$ \\
\hline \multicolumn{5}{|c|}{ Temporal and extratemporal procedures } \\
\hline \multicolumn{5}{|c|}{ All operated patients } \\
\hline Normal neuroimaging & 19 & $14(73.7 \%)$ & $5(26.3 \%)$ & \multirow{2}{*}{$\chi^{2}=0.0026, p=0.96$} \\
\hline Abnormal neuroimaging & 93 & $68(73.1 \%)$ & $25(29.9 \%)$ & \\
\hline \multicolumn{5}{|c|}{ Patients with intracranial electrodes } \\
\hline Normal neuroimaging & 19 & $14(73.7 \%)$ & $5(26.3 \%)$ & \multirow{2}{*}{$\chi^{2}=0.21, p=0.65$} \\
\hline \multirow[t]{2}{*}{ Abnormal neuroimaging } & 34 & $23(67.6 \%)$ & $11(32.3 \%)$ & \\
\hline & $\mathbf{n}$ & Favourable outcome (I-II) & Poor outcome (III-IV) & $\mathrm{F}, \mathrm{p}$ \\
\hline \multicolumn{5}{|l|}{$\begin{array}{l}\text { Temporal procedures } \\
\text { All operated patients }\end{array}$} \\
\hline Normal neuroimaging & 13 & 12 (92.3\%) & $1(7.7 \%)$ & \multirow{2}{*}{$F, p=0.44$} \\
\hline Abnormal neuroimaging & 70 & $56(80 \%)$ & $14(20 \%)$ & \\
\hline \multicolumn{5}{|c|}{ Patients with intracranial electrodes } \\
\hline Normal neuroimaging & 13 & $12(92.3 \%)$ & $1(7.7 \%)$ & \multirow{2}{*}{$F, p=0.63$} \\
\hline \multirow[t]{2}{*}{ Abnormal neuroimaging } & 22 & 18 (81.8\%) & $4(22.2 \%)$ & \\
\hline & n & Favourable outcome (I-II) & Poor outcome (III-IV) & $F, p$ \\
\hline \multicolumn{5}{|c|}{ Extratemporal procedures (including temporo-occipital resections) } \\
\hline \multicolumn{5}{|c|}{ All operated patients } \\
\hline Normal neuroimaging & 6 & $2(33 \%)$ & $4(66 \%)$ & \multirow{2}{*}{$F, p=0.65$} \\
\hline \multicolumn{4}{|c|}{ Patients with intracranial electrodes } & \\
\hline Normal neuroimaging & 6 & $2(33.3 \%)$ & $4(66.7 \%)$ & \multirow{2}{*}{$F, p=0.99$} \\
\hline Abnormal neuroimaging & 12 & $5(41.7 \%)$ & $7(58.3 \%)$ & \\
\hline
\end{tabular}

temporo-occipital resections). For analysis, the two patients who had temporo-occipital resections were considered as extratemporal.

All patients with normal neuroimaging were assessed with intracranial electrodes.

Used electrodes were disposed of to prevent transmission of Creutzfeld-Jacob disease. Prophylactic antibiotics were prescribed (intravenous cefuroxime, $750 \mathrm{mg}$ three times daily) during the period of recording with intracranial electrodes to minimise the risk of infection.

Resections were guided by preoperative subacute intracranial recordings or intraoperative electrocorticography and by intraoperative image guidance (Stealth, Medtronic, Houston, Texas, USA).

\section{Indications for implantation of intracranial electrodes} The following two patient groups had surgery without studies with intracranial electrodes.

Lesionectomy - Patients with a discrete cerebral lesion demonstrated by neuroimaging at a non-functionally eloquent site, with location concordant with seizure semiology, topography of interictal discharges, topography of ictal onset on the scalp EEG if known, distribution of background abnormalities in the interictal EEG, and neuropsychological findings.

Temporal lobectomy-Patients with a consistent single temporal site of seizure onset on scalp EEG telemetry, at or preceding clinical semiology, concordant with seizure semiology, distribution of background abnormalities in the interictal scalp EEG, neuroimaging, and neuropsychological findings.

Patients not fulfilling these criteria had studies with intracranial electrodes. These were patients in whom a hypothesis was available to explain findings to date, particularly any non-convergence of evidence from different tests, and this hypothesis was testable with intracranial electrode implantation. The choice and placement of intracranial electrodes depended on the working hypothesis with regard to the site of seizure onset. As intracerebral (depth) electrodes are perceived to be more invasive that subdural recordings, the latter were generally preferred if possible.
When temporal lobe seizures were suspected but laterality was uncertain, recordings with bilateral 8-contact subtemporal strips inserted through frontotemporal burr holes were carried out. If this procedure yielded inconclusive results, a second intracranial recording was undertaken with bilateral temporal intracerebral electrodes implanted using a lateral approach. When seizures where thought to arise from the frontal lobes, but laterality was uncertain, bilateral intracerebral electrodes were used. When the seizures were thought to arise from the cerebral convexity, from the paracentral lobule, or from the supplementary motor area, mats or strips were used, usually implanted unilaterally.

Surgery was excluded in the following circumstances:

- the EEG showed predominantly generalised interictal EEG discharges in the absence of a discrete lesion on neuroimaging;

- independent sites of electrographic seizure onset were demonstrated in more than one lobe;

- generalised discharges were seen at or immediately preceding clinical seizure onset;

- a site of seizure onset was identified which could not be resected without unacceptable complications and was unsuitable for multiple subpial transection;

- bilateral or multilobar seizure onset was seen with intracranial recordings and there was no clear alternative hypothesis for further studies with intracranial recordings.

\section{Intracranial recordings}

Subdural or intracerebral (depth) electrodes (supplied by AdTech Medical Instruments, Wisconsin, USA) were implanted in all 105 patients assessed with intracranial electrodes.

Subdural electrodes were either strips or mats. Each strip consisted of a single row of four to eight platinum disk electrodes spaced at $10 \mathrm{~mm}$ between centres. The disks were embedded in a $0.7 \mathrm{~mm}$ thick polyurethane strip which overlapped the edges, leaving a diameter of $2.3 \mathrm{~mm}$ exposed, and recessed approximately $0.1 \mathrm{~mm}$ from the surface plane. 
Mats contained rectangular arrays of 12, 16, 20, 32, or 64 similar platinum electrodes.

Intracerebral (depth) electrodes consisted of multicontact flexible bundles of electrodes, which were implanted stereotactically under MRI guidance. The electrodes consisted of six to 10 cylindrical $2.3 \mathrm{~mm}$ long platinum contacts separated by $5 \mathrm{~mm}$ between centres of adjacent electrodes of the same bundle.

Nine patients had depth electrodes in temporal structures only, five had depth electrodes in extratemporal regions only, 21 had temporal and extratemporal depth electrodes, 31 had subdural temporal strips, nine had subdural extratemporal strips, 29 had subdural temporal and extratemporal electrodes, and one had subdural and depth electrodes in temporal and extratemporal regions.

\section{Neuroimaging}

All patients had cerebral MRI. The MRI protocol included the following MRI sequences, which were used in all patients: (1) coronal fast spin echo T2 weighted (time of echo $(\mathrm{TE})=85 \mathrm{~ms}$, time of repetition $(\mathrm{TR})=4300 \mathrm{~ms}), 3.5 \mathrm{~mm}$ slice thickness, $0.5 \mathrm{~mm}$ gap, perpendicular to temporal horn; (2) coronal FLAIR (fluid attenuated inversion recovery) $(\mathrm{TE}=115 \mathrm{~ms}, \quad \mathrm{TR}=8500 \mathrm{~ms}$, inversion time $(\mathrm{TI})=1900 \mathrm{~ms})$, $3.5 \mathrm{~mm}$ slice thickness, $0.5 \mathrm{~mm}$ gap, perpendicular to temporal horn; (3) coronal IR prepped SPGR, $\mathrm{T} 1$ weighted ( $\mathrm{IR}=$ inversion recovery, $\mathrm{SPGR}=$ spoiled gradient recalled), flip angle $30^{\circ}, \mathrm{TE}=2.8 \mathrm{~ms}, \mathrm{TR}=14 \mathrm{~ms}, 1.5 \mathrm{~mm}$ partition; (4) axial fast spin echo $\mathrm{T} 2$ weighted (TE $=75 \mathrm{~ms}$, $\mathrm{TR}=3500 \mathrm{~ms}$ ), $5 \mathrm{~mm}$ slice thickness, $2 \mathrm{~mm}$ gap, parallel to AC-PC line.

Hippocampal volumetry was not used regularly, as it is not widely accepted as routine clinical practice and visual assessment by experienced radiologists appears to be almost as good in detecting hippocampal atrophy. ${ }^{12}$

Unclear non-specific changes of dubious significance were not considered as lesions. Thus patients showing only such changes were included in the group of normal neuroimaging. In equivocal cases, images were reformatted on an Advantage Windows workstation (GE Medical systems).

Computed tomography (CT) was not done routinely. Of the 136 surgically treated patients, 27 had CT, mainly to assess whether there was calcification in the lesion shown on MRI, or to check the position of intracranial electrodes or whether there was a haemorrhage as a complication of the implantation.

\section{Neuropathology}

All resected specimens were fixed in formalin and serially sliced. The slices were processed to paraffin. In specimens with no macroscopic abnormality, all the slices were processed. When a macroscopic lesion was noted, blocks were taken from the lesion, from regions adjacent to the lesion, and from the margins of the specimen. Sections were stained with haematoxylin and eosin, luxol fast blue/cresyl violet, and the silver impregnation method of Glees and Marsland, and immunocytochemistry was carried out by the ABC method for glial fibrillary acidic protein (Dako polyclonal, 1:1250) and neurofilament 200KD (Dako monoclonal, 1:800). Archived slides from all the patients were reviewed.

\section{Surgical outcome}

Surgical outcome with regard to seizure control was determined at regular postoperative follow up assessments by GA and RS. Surgical outcome was classified in four grades according to the following criteria, which are largely based on Engel's classification $^{13}$ : grade I, free of disabling seizures; grade II, almost seizure-free (three or fewer diurnal or nocturnal seizures per year); grade III, worthwhile improvement (but more than three diurnal or nocturnal seizures per year); grade IV, no significant improvement. For analysis, grades I and II were considered a favourable surgical outcome, while grades III and IV were considered poor.

\section{Statistical analysis}

Two tailed $\chi^{2}$ testing with one degree of freedom was used to compare the proportion of patients with favourable outcome between the groups of patients with normal neuroimaging and with abnormal neuroimaging findings. When there were expected frequencies below 5, two tailed Fisher's exact test was used. Existence of significant differences was assumed if at $\mathrm{p}<0.05$. A statistical trend was assumed at $\mathrm{p}>0.05$ but $<0.2$.

\section{RESULTS}

\section{Proportion of patients with normal neuroimaging}

Among the 136 patients operated on, 21 (15\%) showed normal neuroimaging (all 21 were assessed with intracranial electrodes). Of these, 19 had a follow up period of 12 months or longer at the time of analysis. One hundred and fifteen patients $(85 \%)$ showed a lesion on neuroimaging and 93 of these had a follow up period of at least 12 months.

Among the 105 patients assessed using intracranial electrodes, 39 (37\%) had normal neuroimaging, whereas 66 $(63 \%)$ showed a lesion on neuroimaging. Among the 39 patients with normal neuroimaging, 21 (54\%) were operated on and 19 had a follow up period of at least 12 months at the time of analysis. Among the 66 patients with a lesion on neuroimaging, $60(91 \%)$ were operated on and 53 had a follow up period of at least 12 months.

\section{Use of intracranial electrodes in patients with normal neuroimaging}

As mentioned above, all patients with normal neuroimaging were assessed with intracranial electrodes, as data from other tests did not suggest a clear single focus unequivocally.

\section{Seizure control after surgery}

Table 1 shows the proportion of patients with each outcome grade for each patient group. Table 2 shows the comparison of the proportion of patients with favourable and poor outcome in each group. Separate analyses were carried out among patients who had temporal and extratemporal procedures.

\section{All procedures}

\section{All operated patients}

Among the 112 operated patients who had a follow up longer than 12 months, 19 had normal neuroimaging and 93 showed abnormalities on neuroimaging. Among the 19 patients with normal neuroimaging, $74 \%$ had a favourable outcome, and among the 93 patients with abnormal neuroimaging, $73 \%$ had a favourable outcome. There were no statistical differences in the proportion of patients with favourable outcome between those with normal and those with abnormal neuroimaging $\left(\chi^{2}=0.0026,1\right.$ degree of freedom, $\mathrm{p}=0.96$ ) (table 2 ).

\section{Patients operated on after assessment with intracranial electrodes}

Among the 53 operated patients assessed with intracranial electrodes who had a follow up longer than 12 months, 19 had normal neuroimaging and 34 showed abnormalities on neuroimaging. Among the 19 patients with normal neuroimaging, $14(74 \%)$ had a favourable outcome, while among the 34 patients with abnormal neuroimaging, 23 (68\%) had a favourable outcome. There were no statistical differences in the proportion of patients with a favourable outcome 


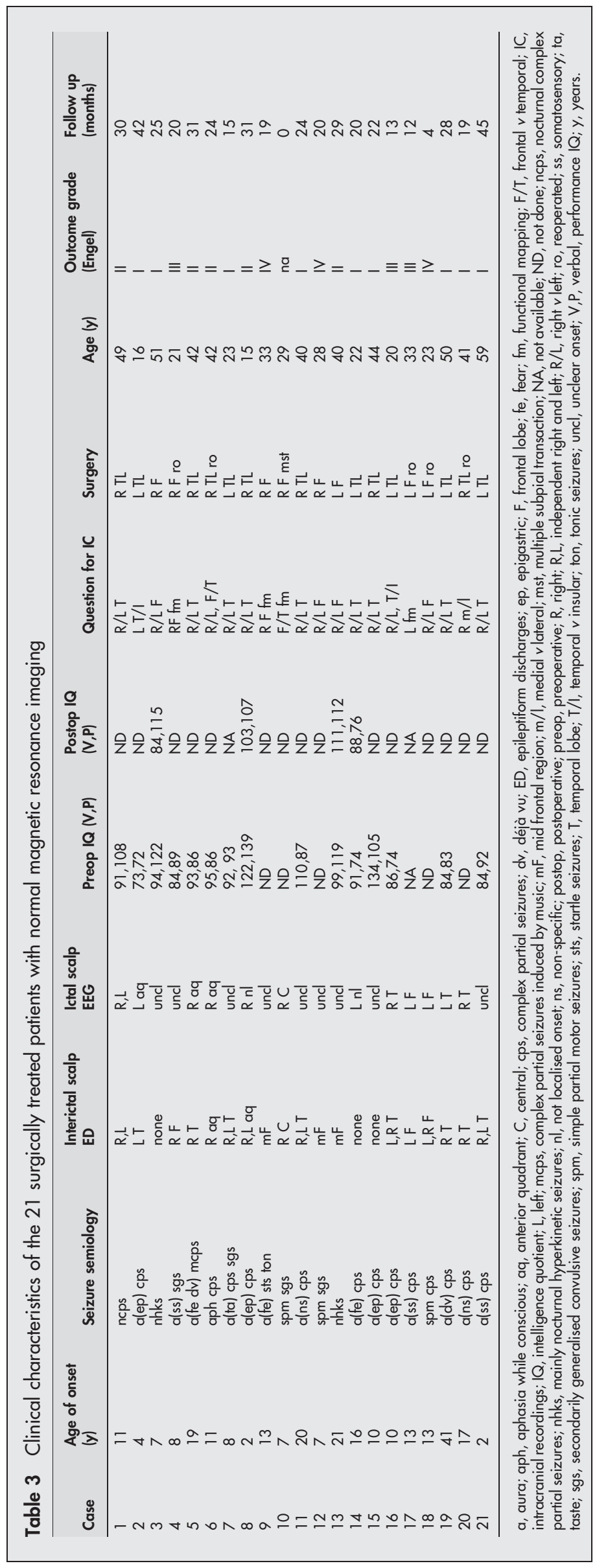


between those with normal and those with abnormal neuroimaging $\left(\chi^{2}=0.21,1\right.$ degree of freedom, $\left.p=0.65\right)$ (table 2).

\section{Temporal resections \\ All operated patients}

Among the 83 patients who had temporal procedures, 13 had normal and 70 had abnormal neuroimaging. Of the 13 with normal neuroimaging, $12(92 \%)$ had a favourable outcome, while among the 70 with abnormal neuroimaging, 56 (80\%) had a favourable outcome. There were no statistical differences in the proportion of patients with a favourable outcome between those with normal and those with abnormal neuroimaging (Fisher's exact test, $\mathrm{p}=0.44$ ) (table 2).

\section{Patients operated on after assessment with intracranial} electrodes

Among the 35 patients who had temporal procedures after being assessed with intracranial electrodes, 13 had normal and 22 had abnormal neuroimaging. Of the 13 with normal neuroimaging, $12(92 \%)$ had a favourable outcome, while among the 22 with abnormal neuroimaging, 18 (82\%) had a favourable outcome. There were no statistical differences in the proportion of patients with favourable outcome between those with normal and those with abnormal neuroimaging (Fisher's exact test, $\mathrm{p}=0.63$ ) (table 2).

\section{Extratemporal procedures (including temporo- occipital resections) \\ All operated patients}

Among the 29 surgically treated patients who had extratemporal procedures after being assessed with intracranial electrodes, six had normal and 23 had abnormal neuroimaging. Of the six patients with normal neuroimaging, two had a favourable outcome, while among the 23 patients with abnormal neuroimaging, $12(52 \%)$ had a favourable outcome. The trend towards a more favourable outcome in patients with abnormal neuroimaging was not significant (Fisher's exact test, $\mathrm{p}=0.65$ ) (table 2).

\section{Patients operated on after assessment with intracranial} electrodes

Among the 18 surgically treated patients who had extratemporal procedures after being assessed with intracranial electrodes, six had normal neuroimaging and 12 had abnormal neuroimaging. Of the six patients with normal neuroimaging, two had a favourable outcome, while among the 12 patients with abnormal neuroimaging, five (42\%) had a favourable outcome. Again, the trend towards more favourable outcome in patients with abnormal neuroimaging was not significant (Fisher's exact test, $\mathrm{p}=0.99$ ) (table 2 ).

Side effects (complications) of intracranial recordings Among the 105 patients studied with intracranial electrodes, five had transitory deficits and two had permanent deficits.

\section{Transitory deficits and complications}

One patient suffered leg weakness for a few days after implantation of parasagittal mats, one developed a subdural haematoma which provoked early removal of mats, one developed a subdural haematoma which provoked removal of haematoma but not of the mats, and two developed infection after removal of subdural electrodes carried out at the same time as the definite surgery, requiring bone flap removal.

Permanent deficits

One patient developed a hemiplegia because of a haematoma after subdural strips, and another patient suffered mild dysphasia resulting from a haemorrhage in the left Sylvian fissure after depth implantation.

\section{Neuropathological findings \\ Patients operated with normal neuroimaging (21 patients)}

Among the 13 patients who had temporal lobe resections, nine showed mesial temporal sclerosis (one had dual pathology with contusional scars), three patients showed non-specific changes, and one had a dysembryoplastic neuroepithelial tumour (DNT). Among the eight patients who had extratemporal surgery (all frontal), three had focal cortical dysplasia, one had a scar from previous surgery, and four showed non-specific changes.

Patients operated with abnormal neuroimaging $(115$ patients)

Among the 88 patients who had temporal lobe resections, 58 showed mesial temporal sclerosis, four of whom had dual pathology: one with Taylor-type focal cortical dysplasia (FCD), one with epidermoid cyst, one with DNT, and one with small scars. Twelve patients had tumours (seven DNT, two gangliogliomas, two astrocytoma grade II, and one oligodendroglioma grade II), six had arteriovenous malformations, two had infarcts, one had non-Taylor-type cortical dysplasia, eight showed non-specific changes, and one had normal neuropathology. Among the 24 patients who had extratemporal surgery, seven showed FCD, eight had tumours (two DNTs, one ganglioglioma, two astrocytomas grade II, one astrocytoma grade III, and two oligodendrogliomas grade II), two had arteriovenous malformations, two had hypothalamic hamartomas, one had an infarct, one had an inflammatory encephalopathy with vascular changes suggestive of an atypical Rasmussen's disease, two had non-specific changes, and in one patient the neuropathological examination was not available as he underwent only multiple subpial transection. Among the three patients who had resections involving temporal and extratemporal structures, one had FCD, one had extensive non-Taylor-type cortical dysplasia in the clinical context of epidermal naevus syndrome, and one patient had a perinatal ischaemic cyst.

Characteristics of patients with normal neuroimaging Table 3 summarises the characteristics of the 21 patients who had normal neuroimaging. These include age at the onset of epilepsy, seizure semiology, findings from interictal and ictal EEG, preoperative and postoperative intelligence quotients (IQ) when done, question to be answered by intracranial recordings, operation type, age at the time of surgery, and outcome and duration of the follow up period. Patient 16 had no seizures during intracranial telemetry. Among the patients who had temporal surgery, patients $1,6,7,8,11,14,15,19$, and 20 showed medial temporal seizure onset, whereas patients 2, 5, and 21 had lateral temporal seizure onset.

\section{DISCUSSION}

Our results suggest that it is worth pursuing surgery in patients with normal neuroimaging. Among patients with temporal resections, there is no difference in seizure control between those with normal and those with abnormal neuroimaging. Regardless of neuroimaging findings, patients with extratemporal procedures clearly show worse overall seizure control than those who underwent temporal resections. Among extratemporal patients, seizure control may be slightly worse in those with normal neuroimaging than in those with neuroimaging abnormalities, although differences were not statistically significant, perhaps because of the smaller sample size. Nevertheless approximately 33\% of extratemporal patients with normal neuroimaging have good 
seizure control. Although no previous studies have directly compared outcome from patients with normal and abnormal neuroimaging, our findings in patients with normal neuroimaging are largely in keeping with those of other investigators..$^{3-8}$

The answer to whether it is worth pursuing surgery in patients with normal MRI requires consideration of several factors apart from seizure control. If intracranial recordings are necessary in these patients, financial issues and the risk of complications from intracranial implantations should be considered. In our series, 81 of 105 patients assessed with intracranial electrodes $(77 \%)$ had surgery, mainly because a focal seizure onset could be found. This proportion was lower among the patients with normal neuroimaging (54\%) than among those with neuroimaging abnormalities (91\%). The likelihood of complications from intracranial recordings is roughly proportional to the number of electrodes implanted. In our series there was a less than $2 \%$ risk of permanent neurological deficit (one patient had hemiplegia and one suffered mild dysphasia, out of 105). Transitory deficits and complications were more common (almost 5\%). Two patients suffered infection of the bone flap after the resective surgery was carried out in the same session as removing chronically implanted subdural electrodes, suggesting that patients are more prone to infection if the final operation is done immediately after chronic subdural recordings. Transmission of Creutzfeldt-Jakob disease has been reported in the past but can be avoided by disposing of used electrodes. Chronic implantation of mats can be associated with leakage of cerebrospinal fluid that can be improved by keeping the head high and changing the head bandage regularly. The risk of complications found in the present series is fairly similar to that reported by other investigators. Mat recordings appear to have a $0.85 \%$ risk of infection, ${ }^{14}$ which can be reduced by prophylactic antibiotics, minimising the duration of implantation, and passing the cables through the scalp at a site remote from the craniotomy. Frank meningitis or encephalitis is rare. Depth electrodes have a very low risk of infection, a $1.9 \%$ risk of haemorrhage with transitory deficits, and a $0.8 \%$ risk of haemorrhage with permanent deficits. ${ }^{15}$

The most common neuropathological abnormality was mesial temporal sclerosis in patients with and without neuroimaging abnormalities, accounting for $50 \%$ and $43 \%$ of patients in those groups, respectively. Presumably the fact that mesial temporal sclerosis may or may not be detected by neuroimaging depends on the severity and extension of neuropathological abnormalities. More sophisticated neuroimaging techniques presently being developed might increase the proportion of patients in whom mesial temporal sclerosis is identified. Patients with normal neuroimaging appear to have a higher proportion of normal or non-specific changes on neuropathological examination (33\% $v$ 8.7\%), which might explain in part their slightly worse outcome with regard to seizure control.

It has been claimed that recent advances in MRI technology will eventually abolish the need for chronic intracranial recordings. This has not been our experience in recent years. As with any diagnostic test, improvements in MRI sensitivity will necessarily be associated with increments in true and false positive detections. In some patients multiple lesions are detected, raising the question of whether there is a single focal seizure onset and which lesion generates seizures. In other patients, mild non-specific unclear abnormalities can be seen, raising doubt about whether they are indeed epileptogenic.

The final decision on whether surgery should be pursued in patients with normal neuroimaging relies on the specific clinical and personal circumstances of each patient. The severity of the patients' epilepsy and its impact on their working and psychosocial background have to be balanced against the chances of improving with surgery and the risk of complications from surgery and from intracranial electrode implantation. We hope that the results reported here will help patients and physicians in taking these difficult decisions.

\section{Authors' affiliations}

G Alarcón, A Valentín, M E Lacruz, R D C Elwes, F Brunhuber, N Mullatti, M Salinas, C D Binnie, Department of Clinical Neurophysiology, King's College Hospital, Denmark Hill, London, UK C Watt, R P Selway, C E Polkey, Department of Neurosurgery, King's College Hospital

J M Jarosz, Department of Neuroimaging, King's College Hospital M Honavar, I Bodi, Department of Neuropathology, King's College Hospital

Competing interests: none declared

\section{REFERENCES}

1 Scott CA, Fish DR, Smith SJ, et al. Presurgical evaluation of patients with epilepsy and normal MRI: role of scalp video-EEG telemetry. J Neurol Neurosurg Psychiatry 1999;66:69-71.

2 Elwes RD, Binnie CD, Polkey CE. Normal magnetic resonance imaging and epilepsy surgery. J Neurol Neurosurg Psychiatry 1999;66:3.

3 Cukiert A, Sousa A, Machado E, et al. Results of surgery in patients with bilateral independent temporal lobe spiking (BITLS) with normal MRI or bilateral mesial temporal sclerosis (MTS) investigated with bilateral subdural grids. Arq Neuropsiquiatr 2000;58:1009-13.

4 Chapman K, Wyllie E, Najm I, et al. Seizure outcome after epilepsy surgery in patients with normal preoperative MRI. I Neurol Neurosurg Psychiatry 2005:76:710-13.

5 Cukiert A, Buratini JA, Machado E, et al. Results of surgery in patients with refractory extratemporal epilepsy with normal or nonlocalizing magnetic resonance findings investigated with subdural grids. Epilepsia 2001;42:889-94

6 Cukiert A, Buratini JA, Machado E, et al. Seizure's outcome after cortical resections including the face and tongue rolandic areas in patients with refractory epilepsy and normal MRI submitted to subdural grids' implantation. Arq Neuropsiquiatr 2001;59:717-21

7 Siegel AM, Jobst BC, Thadani VM, et al. Medically intractable, localizationrelated epilepsy with normal MRI: presurgical evaluation and surgical outcome in 43 patients. Epilepsia $2001 ; 42: 883-8$.

8 Roberts DW, Jobst BC, Siegel AM, et al. Investigation of extra-temporal epilepsy. Stereotact Funct Neurosurg 2001;77:216-18.

9 Ferrier $\mathrm{CH}$, Engelsman J, Alarcon G, et al. Prognostic factors in presurgical assessment of frontal lobe epilepsy. J Neurol Neurosurg Psychiatry 1999;66:350-6.

10 Mosewich RK, So EL, O'Brien TJ, et al. Factors predictive of the outcome of frontal lobe epilepsy surgery. Epilepsia 2000;41:843-9.

11 Elwes RDC. Invasive neurophysiological evaluation. In:Oxbury J, Polkey CE, Duchowny M, editors. Intractable focal epilepsy. London: WB Saunders, 2000:595-615.

12 Jackson GD, Briellmann RS, Kuzniecky RI. Temporal lobe epilepsy. In:Kuzniecky RI, Jackson GD, editors. Magnetic resonance in epilepsy, 2nd ed. Amsterdam: Elsevier Academic Press, 2005:117.

13 Engel J, Van Ness P, Rasmussen T, et al. Outcome in respect to epileptic seizures. In:Engel J, editor. Surgical treatment of the epilepsies. New York: Raven Press, 1993:609-21.

14 Wyler AR, Walker G, Somes G. The morbidity of long-term seizure monitoring using subdural strip electrodes. J Neurosurg 1991;74:734-7.

15 Van Buren JM. Complications of surgical procedures in the treatment and diagnosis of epilepsy. In:Engel J, editor. Surgical treatment of the epilepsies. New York: Raven Press, 1987:465-75. 